\title{
Clinical outcome of patients with metastatic melanoma of unknown primary in the era of novel therapy
}

\author{
Danielle Verver ${ }^{1}$ (D) Dirk J. Grünhagen ${ }^{1}$ - Alexander C. J. van Akkooi ${ }^{2} \cdot$ Maureen J. B. Aarts $^{3}$. \\ Franchette W. P. J. van den Berkmortel ${ }^{4}$. Alfonsus J. M. van den Eertwegh ${ }^{5}$. Jan Willem B. de Groot ${ }^{6}$. \\ Marye J. Boers-Sonderen ${ }^{7}$. John B. A. G. Haanen ${ }^{8}$. Geke A. P. Hospers ${ }^{9}$. Ellen Kapiteijn ${ }^{10}$. Djura Piersma ${ }^{11}$. \\ Rozemarijn S. van Rijn ${ }^{12} \cdot K_{\text {Karijn P. M. Suijkerbuijk }}{ }^{13}$. Albert J.ten Tije ${ }^{14} \cdot$ Gerard Vreugdenhil $^{15}$. Cornelis Verhoef ${ }^{1}$. \\ Astrid A. M. van der Veldt ${ }^{16}$
}

Received: 23 November 2020 / Accepted: 20 January 2021 / Published online: 27 March 2021

(c) The Author(s) 2021

\begin{abstract}
Melanoma of unknown primary (MUP) is considered different from melanoma of known primary (MKP), and it is unclear whether these patients benefit equally from novel therapies. In the current study, characteristics and overall survival (OS) of patients with advanced and metastatic MUP and MKP were compared in the era of novel therapy. Patients were selected from the prospective nation-wide Dutch Melanoma Treatment Registry (DMTR). The following criteria were applied: diagnosis of stage IIIc unresectable or IV cutaneous MKP (cMKP) or MUP between July 2012 and July 2017 and treatment with immune checkpoint inhibition and/or targeted therapy. OS was estimated using the Kaplan-Meier method. The stratified multivariable Cox regression model was used for adjusted analysis. A total of 2706 patients were eligible including 2321 (85.8\%) patients with cMKP and 385 (14.2\%) with MUP. In comparative analysis, MUP patients more often presented with advanced and metastatic disease at primary diagnosis with poorer performance status, higher LDH, and central nervous system metastases. In crude analysis, median OS of cMKP or MUP patients was 12 months (interquartile range [IQR] 5 - 44) and 14 months (IQR 5 - not reached), respectively $(P=0.278)$. In adjusted analysis, OS in MUP patients was superior (hazard rate 0.70 , $95 \%$ confidence interval $0.58-0.85 ; P<0.001)$. As compared to patients with advanced and metastatic cMKP, MUP patients have superior survival in adjusted analysis, but usually present with poorer prognostic characteristics. In crude analysis, OS was comparable indicating that patients with MUP benefit at least equally from treatment with novel therapies.
\end{abstract}

Keywords Melanoma $\cdot$ Unknown primary $\cdot$ Known primary $\cdot$ Novel therapy

$\begin{array}{ll}\text { Abbreviations } \\ \text { CNS } & \text { Central nervous system } \\ \text { cMKP } & \text { Cutaneous melanoma known primary } \\ \text { CTLA-4 } & \text { Cytotoxic T-lymphocyte-associated protein } 4 \\ \text { DMTR } & \text { Dutch Melanoma Treatment Registry } \\ \text { ECOG } & \text { Eastern Cooperative Oncology Group } \\ \text { ICI } & \text { Immune checkpoint inhibition } \\ \text { IQR } & \text { Interquartile range } \\ \text { LDH } & \text { Lactate dehydrogenase } \\ \text { MKP } & \text { Melanoma known primary } \\ \text { MUP } & \text { Melanoma unknown primary } \\ \text { OS } & \text { Overall survival } \\ \text { PD-1 } & \text { Programmed death-1 }\end{array}$

Danielle Verver

d.verver@erasmusmc.nl

Extended author information available on the last page of the article

\section{Introduction}

Melanoma of unknown primary (MUP) is rare, as only 3\% of all melanoma patients present with stage I-IV MUP [1]. Patients with MUP usually present with (presumed) locoregional melanoma metastases in the (sub)cutis, soft tissue, and/or lymph nodes (i.e. stage III disease) or with distant metastases including visceral metastases (i.e. stage IV disease) [2].

To date, the origin of MUP has still not been unravelled. Possible explanations include unrecognised melanomas, (traumatically) removed melanomas without pathological review, the development of de novo melanomas within lymph nodes and/or at other non-cutaneous sites, and missed diagnosis of spontaneous regressing melanomas $[3,4]$. This latter explanation is supported by several studies observing regressed pigmented lesions in patients with MUP [5-9]. 
Spontaneous regression, especially partial spontaneous regression, is rather common in melanoma [[10]. As an enhanced immune response with an increased number of tumour infiltrating $\mathrm{T}$ lymphocytes can be found in regressing melanoma, spontaneous regression is considered the result of an effective host immune response [11,12]. Although the prognostic significance of melanoma regression remains controversial [13], it seems to be associated with favourable prognosis[12]. As MUP may originate from primary melanomas with immune-mediated spontaneous regression, MUP may have a different biology with immunological surveillance mechanisms. As a result, patients with MUP may have a more favourable prognosis as compared to patients with melanoma of known primary (MKP). This hypothesis is supported by a meta-analysis which was conducted before the introduction of novel therapies. In this meta-analysis, patients with stage IV MUP had improved overall survival (OS) as compared with patients with stage IV MKP [14].

Survival of patients with advanced and metastatic MKP and MUP has significantly improved since the introduction of novel therapies, including immune checkpoint inhibition (ICI) and targeted therapy $[1,15]$. Immune checkpoint inhibitors are monoclonal antibodies that enhance anti-tumour T-cell-mediated immune responses by releasing their suppression by immune-checkpoints like cytotoxic T-lymphocyte-associated protein 4 (CTLA-4; e.g. ipilimumab) $[16,17]$ or programmed death-1 (PD-1) receptor (e.g. nivolumab, pembrolizumab) [18-21]. For the treatment of advanced and metastatic melanoma, monotherapy anti-PD1 and combination therapy with ipilimumab and nivolumab has also been approved[22]. Targeted therapy has a different mechanism of action and blocks cancer cell proliferation by selective BRAF inhibitors (BRAFi, i.e. vemurafenib, dabrafenib, encorafenib) and MEK inhibitors (MEKi, i.e. trametinib, cobimetinib, binimetinib) [23-29]. Approximately half of the patients with cutaneous melanoma have benefit from these targeted therapies, which is determined by the presence of a tumour mutation at codon V600 of the BRAF gene. However, targeted therapies may have a more extensive mechanism of action, as these agents are also known to induce immune responses [30].

Until now, information on survival outcomes in patients with MUP treated with these novel therapies is lacking, as clinical trials have not reported on patients with MUP specifically, although they might have been included. Based on the immunological surveillance hypothesis, patients with MUP may derive more benefit from these novel therapies, in particular, since both ICI and targeted therapy have the potential to enhance the anti-tumour immune response [30]. In the current study, patients' characteristics and OS were compared between MUP and MKP patients treated with novel therapies within a large nation-wide prospective Dutch cohort.

\section{Methods}

\section{Data}

Data were retrieved from the Dutch Melanoma Treatment Registry (DMTR), a population-based registry that was initiated in July 2013 to assess the quality of melanoma care in the Netherlands. In the DMTR, safety and efficacy of novel therapies are monitored in real-world clinical practice. Prospective registration started from July 2013. Between July 2012 and July 2013, data were collected retrospectively. The DMTR documents detailed information on all Dutch patients with stage IIIc ${ }^{\text {unresectable }}$ or IV melanoma (advanced and metastatic melanoma), including tumour and patient characteristics, treatment patterns, and clinical outcomes. A detailed description of the DMTR has been published previously [31].

\section{Patients}

For inclusion in the analysis, patients had to fulfil the following inclusion criteria: age $>18$ years, MUP or cutaneous MKP (cMKP), diagnosis of stage IIIc unresectable or IV melanoma between July 2012 and July 2017, and treatment with novel systemic therapy (i.e. ICI and/or targeted therapy) during any of the registered treatment episodes. Melanoma with regional and/or distant metastasis without a primary melanoma was categorised as MUP. Novel systemic therapy included: BRAFi, BRAFi plus MEKi, anti-CTLA-4 monotherapy (ipilimumab), antiPD1 monotherapy (nivolumab or pembrolizumab), and combination therapy of ipilimumab and nivolumab. Data on pre-novel therapy (i.e. other treatment after diagnosis of stage IIIc unresectable or IV melanoma and prior to initiation of novel systemic therapy) were also collected and included local therapy (i.e. surgery and radiotherapy) or other systemic therapy (e.g. chemotherapy). Treatment other than novel systemic therapy prior to diagnosis of stage IIIc unresectable or IV disease, or after initiation of novel systemic therapy were not included in the analyses. Cut-off of follow-up data was set at April 1st 2018.

According to time interval, melanoma was categorised into primary advanced and metastatic disease (i.e. diagnosis of stage IIIc unresectable or IV melanoma $\leq 3$ months after first pathological melanoma diagnosis) and secondary advanced and metastatic disease (i.e. diagnosis of stage IIIc unresectable or IV melanoma $>3$ months after first pathological diagnosis of melanoma). In addition, melanoma specific mutations were categorised into BRAF V600E/K mutation present, absent or unknown. Other BRAF mutations (i.e. non-BRAF V600E/K) were 
categorised as absent. The following patients' and disease characteristics were collected at initiation of first-line novel therapy: age, Eastern Cooperative Oncology Group (ECOG) performance status, number of metastatic sites, central nervous system (CNS) metastases, and serum lactate dehydrogenase (LDH).

As most benefit was expected from ICI, subgroup analyses were performed for patients ever treated with anti-PD1 therapy (including monotherapy and combination with antiCTLA). In addition, survival analyses were performed for patients treated with BRAF inhibitors (BRAFi), BRAFi plus MEK inhibitors (MEKi), ipilimumab monotherapy, anti-PD1 monotherapy, or combination therapy with ipilimumab and nivolumab. Treatment strategy was categorised as first-line therapy ('first'), only line ('only'), and at any time ('ever').

\section{Outcomes}

The primary outcome measure was OS. The OS time was defined from start date of first-line novel therapy to last date of follow-up or death by any cause.

\section{Statistical analysis}

Data were presented as prevalence (percentage) or median (interquartile range [IQR]). Differences between groups were calculated using chi-square tests, Fisher exact tests or non-parametric Mann-Whitney $U$ tests. Among survivors, the median duration of follow-up was calculated from date of initiation of novel therapy to date of last followup using the reversed Kaplan-Meier method (deaths were censored). Crude (unadjusted) OS was estimated using the Kaplan-Meier method and presented in median with IQR. The log rank test was used to compare survival. Only available data were analysed with listwise deletion in multivariable analysis. For adjusted analysis, a multivariable Cox proportional hazards regression analysis was used to assess the effect of several potential prognostic factors on OS. Based on literature review and availability of sufficient data, the following variables were identified as potential prognostic factors: gender, origin of melanoma (cMKP or MUP), timing of metastasis (i.e. primary versus secondary advanced and metastatic disease), BRAF V600E/K mutation status in melanoma, pre-novel therapy (i.e. treatment other than novel systemic therapy initiated after diagnosis of advanced and metastatic disease and prior to initiation of novel systemic therapy), clinical characteristics at start of novel therapy (age, ECOG performance status, serum LDH level, CNS metastases), and treatment with anti-PD1 therapy (i.e. monotherapy and combination) [2,3,32-36]. The proportional hazards assumption was tested by correlating the corresponding set of scaled Schoenfeld residuals with time, thereby testing for independence between residuals and time. For variables affecting the proportional hazards assumption, the stratified Cox procedure was used. $P$ values $\leq 0.05$ were considered statistically significant. All statistical analyses were performed using SPSS version 25.0 (IBM, Armonk, NEW York, USA) and R (version 3.6.1, R Foundation for Statistical Computing, Vienna, Austria, 2019).

\section{Results}

\section{Patient characteristics}

Between July 2012 and July 2017, 3903 patients (age $\geq 18$ years) with advanced and metastatic melanoma were registered in the Netherlands. After exclusion, a total of 2706 out of 3903 patients were eligible for the current study including 2321 patients with cMKP $(85.8 \%)$ and 385 patients with MUP (14.2\%) (Fig. 1). For all survivors, the median follow-up was 24 months (IQR 14 - 35).

At primary diagnosis, patients with MUP more often presented with advanced and metastatic disease (i.e. stage IIIc ${ }^{\text {unresectable }}$ or IV melanoma) as compared with patients with cMKP $(72.5 \%$ versus $7.3 \%$, respectively, $P<0.001)$. In addition, patients with MUP more frequently presented with significantly worse ECOG performance status and CNS metastases (Table 1). BRAF V600E/K mutation was present in $59.2 \%$ and $54.3 \%$ of patients with cMKP and MUP, respectively $(P=0.038)$. Among patients with cMKP and MUP, pre-novel therapy was significantly different $(P=0.043)$, as more patients with cMKP received systemic therapy (e.g. chemotherapy; $5.6 \%$ versus $2.9 \%$ ) (Table 1 ).

\section{Novel therapy}

Time from diagnosis of stage IIIc unresectable and IV melanoma to initiation of first-line novel therapy was not different between MUP and cMKP patients ( 1 month (IQR $0-2$ ) and 1 month (IQR $0-2$ ), respectively, $P=0.444$ ). Applied novel therapy strategies (first, only, and ever) for both ICI and targeted therapy, as well as the total number of novel therapy lines were similar for patients with MUP and cMKP (Table 1). Overall, 1150 patients with cMKP (49.5\%) and 196 patients with MUP (50.9\%) ever received anti-PD1 therapy for stage IIIc unresectable or IV melanoma. In this subgroup, BRAF V600E/K mutation was comparable in cMKP and MUP patients $(46.6 \%$ vs. $51.0 \%, \mathrm{P}=0.114)$, whereas patients with MUP more frequently presented with advanced and metastatic disease at primary diagnosis with worse ECOG performance status, higher LDH, and CNS metastases (Table 1). 
Fig. 1 Flow diagram of patient selection

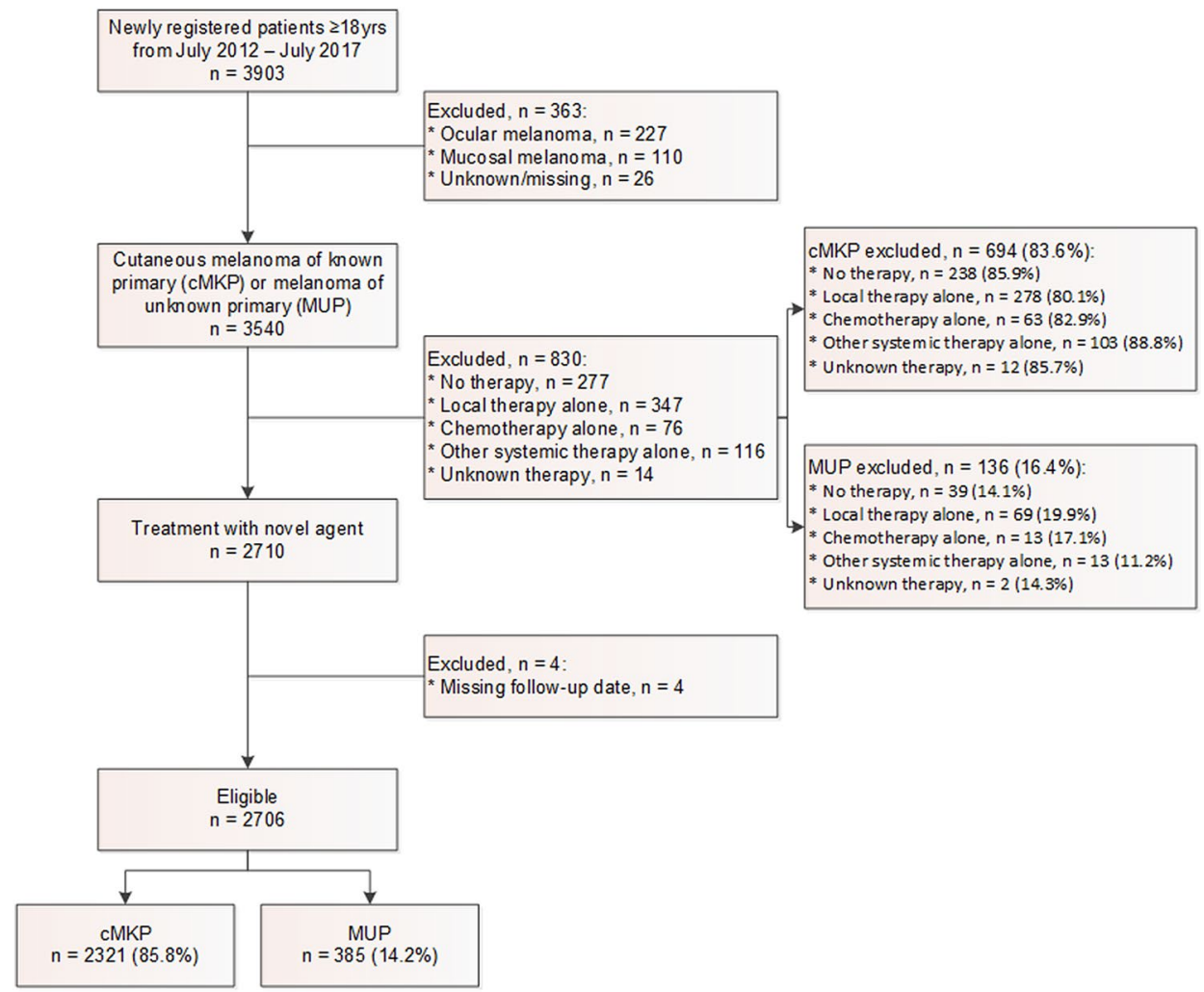

\section{Survival}

In crude analysis, patients with cMKP and MUP had comparable median OS of 12 months (IQR, $5-44$ ) and 14 months (IQR, 5 - not reached), respectively ( $P=0.28$; Fig. 2a). In the subgroup of patients ever treated with anti-PD1 therapy for stage IIIc unresectable or IV, a comparable median OS of 27 months (IQR, 10 - 56) and 26 months, (IQR 10 - not reached) was measured in patients with cMKP and MUP $(\mathrm{P}=0.52)$, respectively (Fig. $2 \mathrm{~b})$. In addition, OS was not different for all other strategies of administered novel therapy (i.e. BRAFi, BRAFi plus MEKi, ipilimumab monotherapy, anti-PD1 monotherapy or ipilimumab + nivolumab) as first, only, and ever treatment line (Fig. 3.).

In multivariable analysis, patients with MUP had improved OS as compared to patients with cMKP, when adjusted for age, gender, CNS metastases, timing of metastasis, pre-novel therapy, and stratified (due to affecting the proportional hazards assumption) for ECOG performance, LDH, BRAF V600E/K mutation, and anti-PD1 therapy (hazard rate $0.74,95 \%$ confidence interval (CI) $0.61-0.90$; $P=0.002$ ) (Table 2). In patients ever treated with anti-PD1 therapy, OS was improved in patients with MUP as compared to patients with cMKP, when adjusted for age, gender, timing of metastasis, ECOG performance status, CNS metastases, pre-novel therapy, ipilimumab combined with nivolumab therapy, and stratified for serum LDH and BRAF
V600E/K mutation (hazard rate $0.87,95 \%$ CI 0.48 - 0.96; $P=0.028$ ) (Table 2). The adjusted expected survival curves of the analyses are shown in Fig. 4.

\section{Discussion}

To date, this study represents the largest study in patients with advanced and metastatic MUP and cMKP in the novel therapy era. For stage IIIc unresectable and IV, survival advantage was measured for patients with MUP as compared to patients with cMKP, when adjusted for several prognostic factors. However, in crude analysis, OS was similar, even in patients ever treated with anti-PD1 therapy.

MUP is considered relatively rare as approximately $3 \%$ of all patients with newly diagnosed melanoma stage I-IV present with MUP [1]. However, MUP is more common in patients with advanced and metastatic melanoma. In the current study, approximately $14 \%$ of all patients with stage IIIc ${ }^{\text {unresectable }}$ and IV melanoma were diagnosed with MUP. These results are supported by similar rates in other reports [37]. We previously demonstrated that the introduction of novel therapies for patients with (primary) advanced and metastatic MUP has led to a significantly improved median OS from 4 to 11 months [1]. The current study focusses on the relevant question whether this survival benefit is similar for patients with MUP as compared to patients with cMKP. To this end, a large nation-wide prospective Dutch cohort 
Table 1 Comparative analysis of patient, disease and treatment characteristics

\begin{tabular}{|c|c|c|c|c|c|c|}
\hline \multicolumn{4}{|l|}{ All patients } & \multicolumn{3}{|c|}{$\begin{array}{l}\text { Anti-PD1 therapy ever (monotherapy and } \\
\text { combination) }\end{array}$} \\
\hline Characteristics & $\operatorname{cMKP}(n=2321)$ & $\operatorname{MUP}(n=385)$ & $P$ & $\mathrm{cMKP}(n=1150)$ & $\operatorname{MUP}(n=196)$ & $P$ \\
\hline Age, yrs & $62(52-71)$ & $61(53-69)$ & 0.108 & $63(53-71)$ & $62(53-69)$ & 0.459 \\
\hline Gender & & & $0.368^{\$}$ & & & $0.378^{\$}$ \\
\hline Male & $1362(58.7)$ & $238(61.8)$ & & $671(58.3)$ & $123(62.8)$ & \\
\hline Female & $958(41.3)$ & $147(38.2)$ & & $478(41.6)$ & $73(37.2)$ & \\
\hline Unknown & $1(0.1)$ & 0 & & $1(0.1)$ & 0 & \\
\hline Timing advanced and metastatic disease & & & $<0.001$ & & & $<0.001$ \\
\hline Primary & $169(7.3)$ & $279(72.5)$ & & $84(7.3)$ & $154(78.6)$ & \\
\hline Secondary & $2152(92.7)$ & $106(27.5)$ & & $1066(92.7)$ & $42(21.4)$ & \\
\hline ECOG performance & & & 0.004 & & & 0.044 \\
\hline 0 & $1191(51.3)$ & $163(42.3)$ & & $680(64.0)$ & $99(54.4)$ & \\
\hline$\geq 1$ & $929(40.0)$ & $186(48.3)$ & & $382(36.0)$ & $83(45.6)$ & \\
\hline Unknown & $201(8.7)$ & $36(9.4)$ & & $88(7.7)$ & $14(7.1)$ & \\
\hline LDH value & & & 0.096 & & & 0.013 \\
\hline Normal & $1428(61.5)$ & $220(57.1)$ & & $767(66.7)$ & $110(56.1)$ & \\
\hline Elevated & $836(36.0)$ & $159(41.3)$ & & $358(31.1)$ & $82(41.8)$ & \\
\hline Unknown & $57(2.5)$ & $6(1.6)$ & & $25(2.2)$ & $4(2.0)$ & \\
\hline CNS metastases & & & 0.001 & & & 0.002 \\
\hline Absent & $1552(66.9)$ & $250(64.9)$ & & $807(70.2)$ & $133(67.9)$ & \\
\hline Present & $595(25.6)$ & $123(31.9)$ & & $254(22.1)$ & $59(30.1)$ & \\
\hline Unknown & $174(7.5)$ & $12(3.1)$ & & $89(7.7)$ & $4(2.0)$ & \\
\hline No. of metastases independent of location & & & 0.836 & & & 0.582 \\
\hline$<5$ & $292(12.6)$ & $52(13.5)$ & & $171(14.9)$ & $27(13.8)$ & \\
\hline $5-10$ & $141(6.1)$ & $26(6.8)$ & & $86(7.5)$ & $11(5.6)$ & \\
\hline$>10$ & $1576(67.9)$ & $260(67.5)$ & & $701(61.0)$ & $129(65.8)$ & \\
\hline Unknown & $312(13.4)$ & $47(12.2)$ & & $192(16.7)$ & $29(14.8)$ & \\
\hline BRAF V600E/K mutation & & & 0.038 & & & 0.114 \\
\hline Absent & $848(36.5)$ & $150(39.0)$ & & $536(46.6)$ & $100(51.0)$ & \\
\hline Present & $1375(59.2)$ & $209(54.3)$ & & $565(49.1)$ & $83(42.3)$ & \\
\hline Unknown & $98(4.2)$ & $26(6.8)$ & & $49(4.3)$ & $13(6.6)$ & \\
\hline Pre-novel therapy ${ }^{@}$ & & & 0.043 & & & 0.232 \\
\hline None & $2020(87.0)$ & $339(88.1)$ & & $993(86.3)$ & $167(85.2)$ & \\
\hline Local therapy (e.g. surgery, radiotherapy) & $170(7.3)$ & $35(9.1)$ & & $113(9.8)$ & $25(12.8)$ & \\
\hline Systemic therapy (e.g. chemotherapy, other) & $131(5.6)$ & $11(2.9)$ & & $44(3.8)$ & $4(2.0)$ & \\
\hline Novel therapy first-line & & & 0.819 & & & 0.966 \\
\hline First-line BRAFi & $711(30.6)$ & $106(27.5)$ & & $113(9.8)$ & $22(11.2)$ & \\
\hline First-line BRAFi + MEKi & $367(15.8)$ & $65(16.9)$ & & $150(13.0)$ & $25(12.8)$ & \\
\hline First-line ipi & $574(24.7)$ & $99(25.7)$ & & $218(19.0)$ & $34(17.3)$ & \\
\hline First-line anti-PD1 mono & $593(25.5)$ & $102(26.5)$ & & $593(51.6)$ & $102(52.0)$ & \\
\hline First-line ipi + nivo & $76(3.3)$ & $13(3.4)$ & & $76(6.6)$ & $13(6.6)$ & \\
\hline Novel therapy only & & & 0.691 & & & 0.897 \\
\hline BRAFi only & $417(18.0)$ & $58(15.1)$ & & $\mathrm{n} / \mathrm{a}$ & $\mathrm{n} / \mathrm{a}$ & \\
\hline BRAFi + MEKi only & $168(7.2)$ & $32(8.3)$ & & $\mathrm{n} / \mathrm{a}$ & $\mathrm{n} / \mathrm{a}$ & \\
\hline Ipi only & $291(12.5)$ & $54(14.0)$ & & $\mathrm{n} / \mathrm{a}$ & $\mathrm{n} / \mathrm{a}$ & \\
\hline Anti-PD1 mono only & $422(18.2)$ & $75(19.5)$ & & $422(36.7)$ & $75(38.3)$ & \\
\hline Ipi + nivo only & $58(2.5)$ & $9(2.3)$ & & $58(5.0)$ & $9(4.6)$ & \\
\hline Novel therapy combinations ${ }^{\wedge}$ & 965 (41.6) & $157(40.8)$ & & $670(58.3)$ & $112(57.1)$ & \\
\hline Novel therapy ever ${ }^{\#}$ & & & & & & \\
\hline
\end{tabular}


Table 1 (continued)

\begin{tabular}{|c|c|c|c|c|c|c|}
\hline \multicolumn{4}{|l|}{ All patients } & \multicolumn{3}{|c|}{$\begin{array}{l}\text { Anti-PD1 therapy ever (monotherapy and } \\
\text { combination) }\end{array}$} \\
\hline Characteristics & $\operatorname{cMKP}(n=2321)$ & $\operatorname{MUP}(n=385)$ & $P$ & $\operatorname{cMKP}(n=1150)$ & $\operatorname{MUP}(n=196)$ & $P$ \\
\hline BRAFi ever & $824(35.5)$ & $121(31.4)$ & 0.120 & $167(14.5)$ & $30(15.3)$ & 0.774 \\
\hline BRAFi + MEKi ever & $586(25.2)$ & $102(26.5)$ & 0.603 & $328(28.5)$ & $55(28.1)$ & 0.895 \\
\hline Ipi ever & 849 (36.6) & $147(38.2)$ & 0.546 & $346(30.1)$ & $60(30.6)$ & 0.882 \\
\hline Anti-PD1 mono ever & $1022(44.0)$ & $178(46.2)$ & 0.421 & $1022(88.9)$ & $196(90.8)$ & 0.418 \\
\hline Ipi + nivo ever & $156(6.7)$ & $23(6.0)$ & 0.585 & $156(13.6)$ & $23(11.7)$ & 0.485 \\
\hline No. of novel therapy lines & & & 0.357 & & & 0.295 \\
\hline One line & $1356(58.4)$ & $228(59.2)$ & & $480(41.7)$ & $84(42.9)$ & \\
\hline Two line & $642(27.7)$ & $93(24.2)$ & & $416(36.2)$ & $59(30.1)$ & \\
\hline Three lines & $215(9.3)$ & $43(11.2)$ & & $159(13.8)$ & $32(16.3)$ & \\
\hline$>$ Three lines & $108(4.7)$ & $21(5.5)$ & & $95(8.3)$ & $21(10.7)$ & \\
\hline
\end{tabular}

BRAFi BRAF inhibition; CNS central nervous system; Ipi ipilimumab; LDH lactate dehydrogenase; MEKi MEK inhibition; Nivo nivolumab;

\$Fisher exact test

${ }^{\circledR}$ After diagnosis of advanced and metastatic disease but prior to initiation of novel therapy

\#percentage yes per category

${ }^{\wedge}$ Concerns patients who received more than one line of novel therapy

was analysed, thereby including both patients with primary and secondary advanced and metastatic disease.

Compared to patients with cMKP, patients with MUP more frequently presented with poorer prognostic factors, including advanced and metastatic melanoma at primary diagnosis, higher ECOG performance status, higher LDH, and CNS metastases. Interestingly, despite these poorer prognostic factors, patients with MUP had comparable OS in crude analysis. This may suggest that patients with MUP have favourable factors which are still unknown. In adjusted analysis, correcting for the known poorer prognostic factors, patients with MUP show improved OS. In another large study, conducted before the introduction of novel therapies, patients with MUP also had similar OS in crude analysis and improved survival in adjusted analysis [38]. These findings suggest that the possible favourable factors in patients with MUP are not affected by novel therapy. Based on the immunological surveillance hypothesis, a larger benefit from novel therapies, especially ICI, may have been expected in patients with MUP thus resulting in improved survival even in the unadjusted analysis.

Our results are supported by a recent Danish study in 576 patients comparing survival between patients with cMKP $(n=496)$ and MUP $(n=80)$ after the introduction of novel therapies [39]. In this Danish analysis, approximately $40 \%$ of the included patients had relatively good prognostic factors including ECOG $0-1$, normal LDH, and absence of active CNS metastases. Patients with MUP showed poorer prognostic factors in terms of disease stage. Nevertheless, OS was comparable in crude analysis, with median OS of 9.7 and 10.0 months for patients with cMKP and MUP
$(P=0.84)$, respectively. The imbalance in disease stage partly explains the non-superior survival for patients with MUP [40]. The observed lower median survival may be explained by the fact that patients who were not treated with novel therapies (or not treated at all) were also included. Another recent small pilot study showed different results in 41 patients treated with ICI [41]. The patient population was small and included a relatively high number of patients with MUP (22\%) with comparable baseline characteristics as patients with MKP. Although this population may not be representative of real-world MUP patients, the pilot study showed an OS benefit in MUP patients treated with ICI.

In clinical trials on novel therapy, outcomes for patients with MUP have not been reported, and it is unclear how many patients with MUP were included. Although MUP was not an exclusion criterion, it is likely that most patients with MUP were ineligible based on other criteria such as ECOG performance status $>1$, elevated $\mathrm{LDH}$, and presence of (symptomatic) CNS metastases. As demonstrated in the current study, patients with MUP at least have similar benefit from treatment with novel agents, despite these poorer prognostic factors.

Overall, patients with cMKP and MUP were treated according to similar strategies. Also, for the excluded patients who did not receive novel therapy the distribution of cMKP and MUP was largely similar (Fig. 1). Noteworthy, approximately half of the patients in both groups ever received anti-PD1 therapy during the course of treatment. This relatively limited use of anti-PD1 therapy is presumably related to the years of approval, availability and/or incorporation in Dutch guidelines. In addition, some patients might 
a

Overall survival

All patients

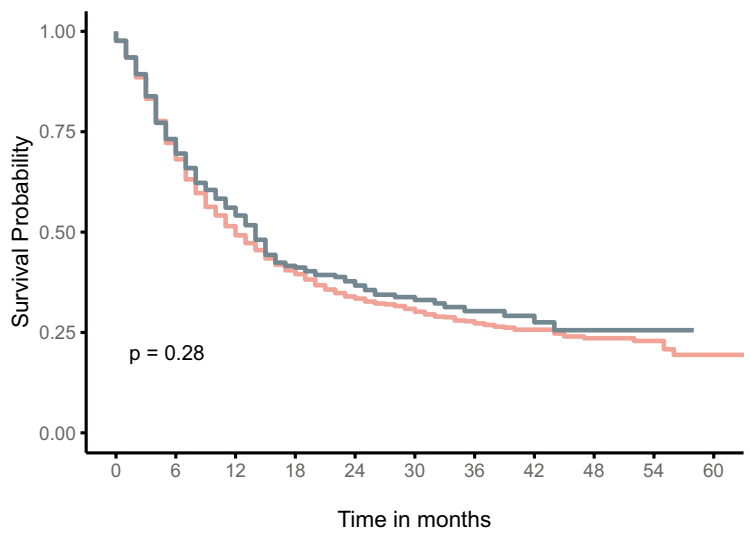

$\begin{array}{lllllllllll}\text { CMKP } 2321 & 1554 & 948 & 590 & 385 & 256 & 153 & 94 & 49 & 24 & 4\end{array}$

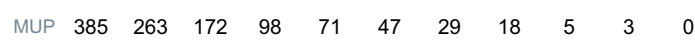

\section{b}

\section{Overall survival}

Anti-PD1 therapy ever

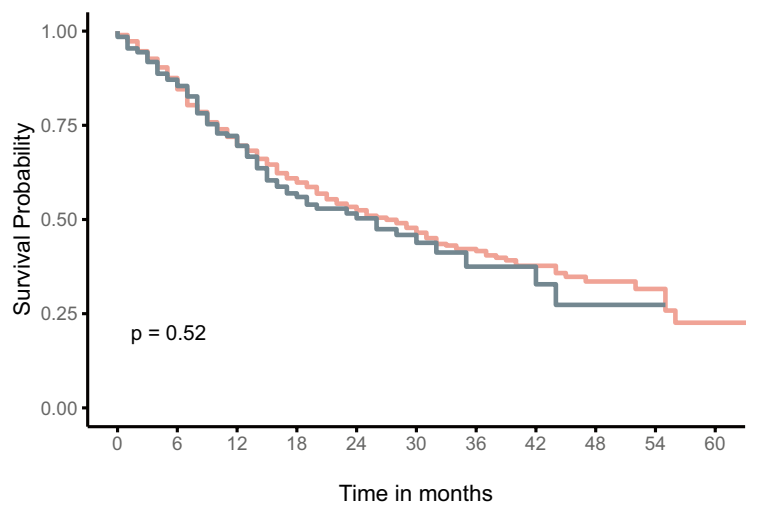

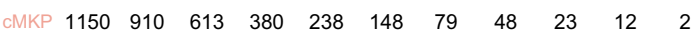

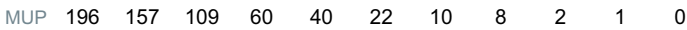

Fig. 2 Crude survival in a all patients and $\mathbf{b}$ patients ever treated with anti-PD1 therapy (monotherapy and combination)

have had long-term benefit from targeted therapy without the need for anti-PD1 therapy, whereas other patients might have had rapid progressive disease while anti-PD1 monotherapy or the combination was not available.
The current study has several limitations of which some are inherently related to the registration of real-world data such as incomplete data. Therefore, it is conceivable that some cases of MUP may have been misclassified, as it was not registered whether patients with MUP had exclusion criteria for diagnosis of MUP, including prior orbital exenteration or enucleation, prior skin excision, electrodessication, cauterization, or other surgical manipulation of a mole, freckle, birthmark, paronychia, or skin blemish [42]. In addition, some MUPs may have been misclassified because of limited diagnostics, since the Dutch Melanoma guidelines do not recommend endoscopy, ophthalmoscopy and/ or nasopharyngoscopy. Unfortunately, information on these examinations was not available in the DMTR. On the other hand, extensive diagnostic imaging is usually performed in patients with MUP, as these patients primarily present with stage IIIb-IV. In the current study, data on positron emission tomography (PET) were only available at the time of initial staging of IIIc unresectable and IV melanoma. As $72.5 \%$ of MUP patients primarily presented with stage IIIcunresectable and IV melanoma and $65.6 \%$ of these patients underwent PET at initial staging, misclassification of MUP is probably limited. Another limitation may be the presence of lead-time bias due to different disease patterns of MUP and cMKP, with advanced and metastatic disease already present at time of primary diagnosis of MUP in most cases. Therefore, it is conceivable that advanced and metastatic disease was detected earlier in patients with cMKP as a result of patient awareness and active surveillance after primary diagnosis of cMKP. Another potential limitation is the limited follow-up period for newer agents, potentially resulting in less representative survival data for patients treated with these agents. Finally, patients with non-cutaneous MKP (i.e. ocular and mucosal primary melanoma) were excluded in order to generate a more homogenous population, although it is unknown whether MUP may have its origin in noncutaneous sites. On the other hand, the genotypes of MUP and cMKP are comparable, indicating that MUP most likely arises from (regressed) cutaneous sites [43-45].

In conclusion, as compared to patients with advanced and metastatic cMKP, patients with MUP have comparable overall survival in crude analysis and show superior survival in adjusted analysis. This indicates that patients with MUP benefit at least equally from treatment with novel therapies, although they usually present with poorer prognostic factors. Therefore, novel therapy should not be withheld in patients with advanced stage MUP. 


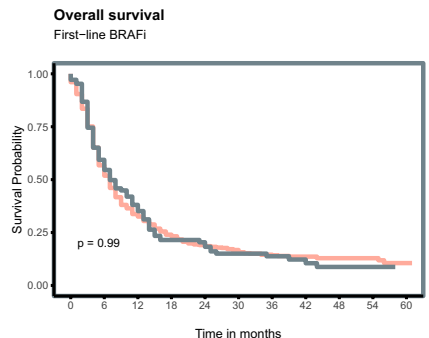

Time in months

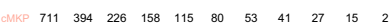

Mup $\begin{array}{lllllllllll}106 & 62 & 39 & 21 & 19 & 14 & 11 & 7 & 2 & 2 & 0\end{array}$ Overall survival

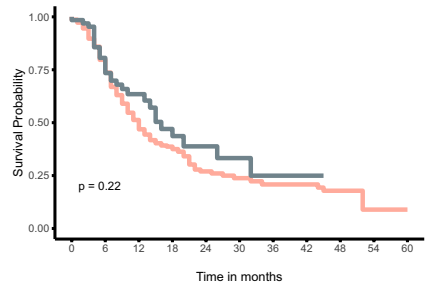
Time in months
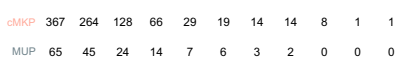

Overall survival

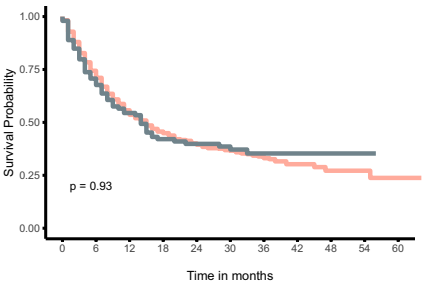

Time in months

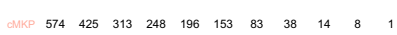

MuP $99 \begin{array}{llllllllll}70 & 53 & 40 & 35 & 27 & 15 & 9 & 3 & 1 & 0\end{array}$

Overall survival

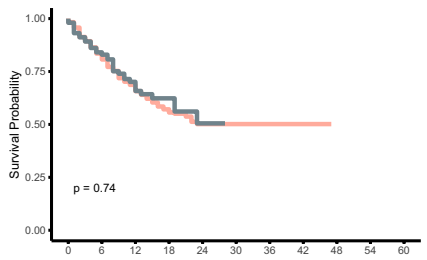

Time in months

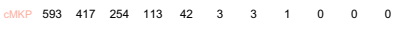

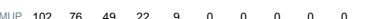

Overall survival

First-line ipilim

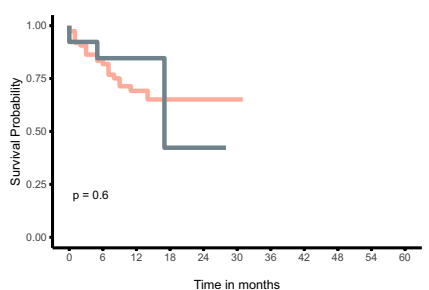

Time in months

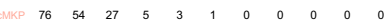

Mup $\begin{array}{lllllllllll}13 & 10 & 7 & 1 & 1 & 0 & 0 & 0 & 0 & 0 & 0\end{array}$

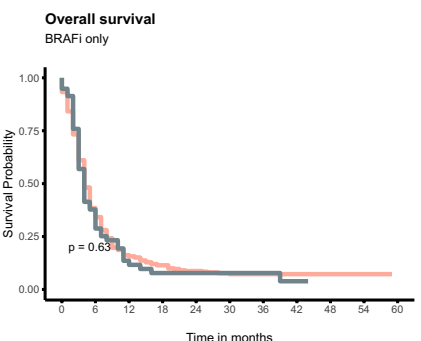

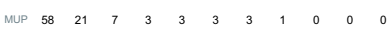

Overall survival

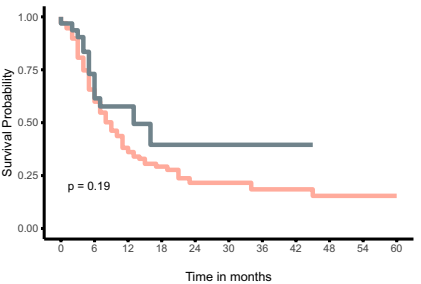

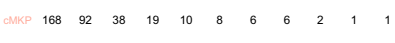

\section{Overall survival}

Ipilimumab only

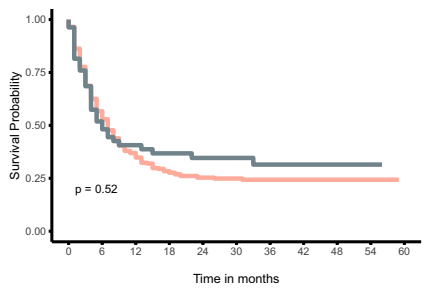

$\begin{array}{lllllllllll}\text { MKP } 291 & 164 & 105 & 76 & 63 & 47 & 31 & 16 & 6 & 3 & 0\end{array}$

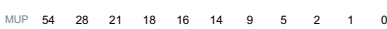

Overall survival

Anti-PD1 monotherapy on

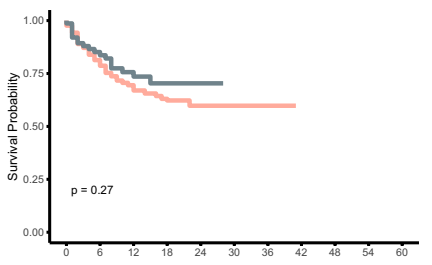

Time in months

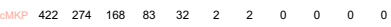

Mup $\begin{array}{lllllllllll}75 & 56 & 36 & 16 & 7 & 0 & 0 & 0 & 0 & 0 & 0\end{array}$

Overall survival

Ipilimumab + nivolumab only

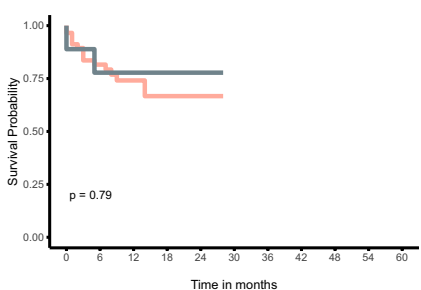

Time in months

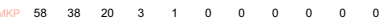

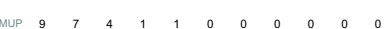

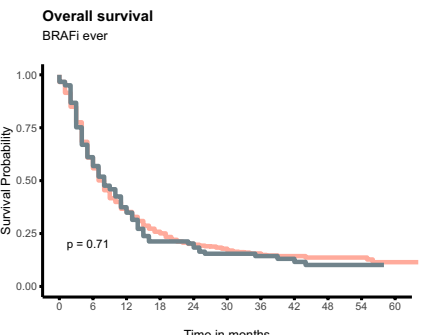

Time in months

$\begin{array}{llllllllllll}\text { CMKP } & 824 & 491 & 286 & 198 & 141 & 102 & 66 & 47 & 31 & 17 & 3\end{array}$

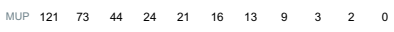

Overall survival

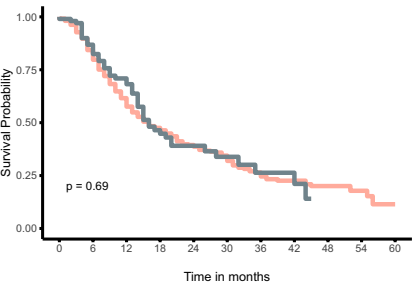

Time in months

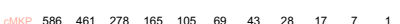

MUP $\begin{array}{lllllllllll}102 & 79 & 52 & 28 & 16 & 11 & 7 & 5 & 0 & 0 & 0\end{array}$

Overall survival

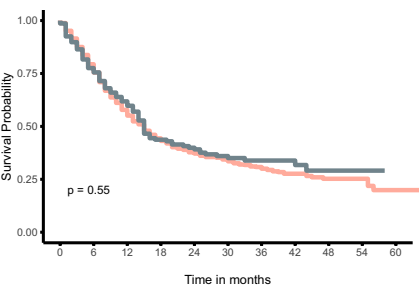

Time in months

$\begin{array}{llllllllllll}\text { UKP } & 849 & 668 & 469 & 336 & 256 & 198 & 116 & 64 & 33 & 16 & 3\end{array}$

MuP $147 \begin{array}{lllllllllll}114 & 89 & 60 & 52 & 38 & 25 & 16 & 5 & 3 & 0\end{array}$

Overall survival

Anti-PD1 monotherapy ever

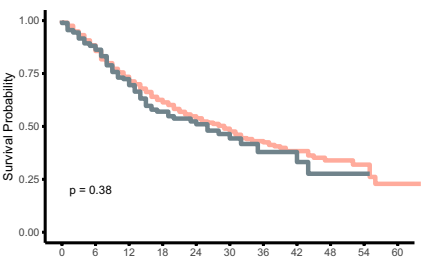

Time in months

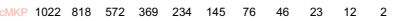

$\begin{array}{llllllllllll}\text { MuP } & 178 & 144 & 101 & 56 & 39 & 22 & 10 & 8 & 2 & 1 & 0\end{array}$

Overall surviva

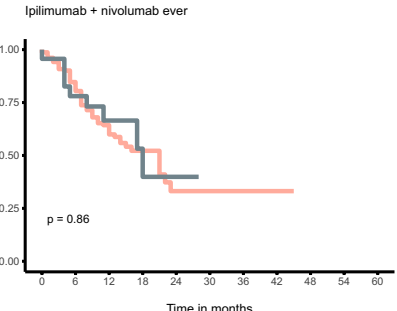

Time in months

$\begin{array}{lllllllllll}156 & 120 & 59 & 19 & 7 & 5 & 3 & 2 & 0 & 0 & 0\end{array}$ $\begin{array}{llllllllllll}\text { MUP } & 23 & 16 & 10 & 4 & 1 & 0 & 0 & 0 & 0 & 0 & 0\end{array}$

Fig. 3 Crude OS in patients with MKP and MUP treated first-line, only or ever with BRAFi monotherapy, BRAFi plus MEKi, ipilimumab monotherapy, anti-PD1 monotherapy, and combination of ipilimumab and nivolumab 
Table 2 Stratified Cox regression models for overall survival for all patients and according to anti-PD1 therapy ever (monotherapy and combination)

\begin{tabular}{|c|c|c|c|c|}
\hline \multicolumn{3}{|l|}{ All patients ${ }^{\mathrm{a}}$} & \multicolumn{2}{|l|}{ Anti-PD1 therapy ${ }^{b}$} \\
\hline & HR $(95 \% \mathrm{CI})$ & $P$ value & HR $(95 \% \mathrm{CI})$ & $P$ value \\
\hline \multicolumn{5}{|l|}{ Origin } \\
\hline cMKP & Reference & & Reference & \\
\hline MUP & $0.74(0.61-0.90)$ & 0.002 & $0.87(0.48-0.96)$ & 0.028 \\
\hline Age, yrs & $1.01(1.00-1.01)$ & 0.017 & $1.01(1.01-1.02)$ & 0.001 \\
\hline \multicolumn{5}{|l|}{ Gender } \\
\hline Male & Reference & & Reference & \\
\hline Female & $0.86(0.77-0.96)$ & 0.010 & $0.97(0.80-1.18)$ & 0.766 \\
\hline \multicolumn{5}{|c|}{ Timing advanced and metastatic disease } \\
\hline Primary & Reference & & Reference & \\
\hline Secondary & $0.84(0.70-1.01)$ & 0.066 & $0.67(0.49-0.91)$ & 0.010 \\
\hline \multicolumn{5}{|c|}{ ECOG performance } \\
\hline 0 & $\mathrm{n} / \mathrm{a}$ & $\mathrm{n} / \mathrm{a}$ & Reference & \\
\hline$>0$ & $\mathrm{n} / \mathrm{a}$ & $\mathrm{n} / \mathrm{a}$ & $1.47(1.21-1.78)$ & $<0.001$ \\
\hline \multicolumn{5}{|l|}{ CNS metastases } \\
\hline No & Reference & & Reference & \\
\hline Yes & $1.65(1.46-1.86)$ & $<0.001$ & $1.71(1.39-2.10)$ & $<0.001$ \\
\hline \multicolumn{5}{|l|}{ Pre-novel therapy ${ }^{c}$} \\
\hline None & Reference & & Reference & \\
\hline Local therapy & $0.88(0.68-1.14)$ & 0.325 & $0.78(0.54-1.13)$ & 0.189 \\
\hline Systemic therapy & $0.94(0.73-1.20)$ & 0.618 & $1.11(0.69-1.78)$ & 0.680 \\
\hline \multicolumn{5}{|l|}{ Ipi + nivo ever } \\
\hline No & $\mathrm{n} / \mathrm{a}$ & $\mathrm{n} / \mathrm{a}$ & Reference & \\
\hline Yes & $\mathrm{n} / \mathrm{a}$ & $\mathrm{n} / \mathrm{a}$ & $1.35(1.01-1.79)$ & 0.040 \\
\hline
\end{tabular}

CNS central nervous system; $n / a$ not applicable

${ }^{a}$ Stratified by serum level LDH, BRAF V600E/K, ECOG performance and anti-PD1 therapy status ${ }^{\mathrm{b}}$ Stratified by serum level LDH and BRAF V600E/K

${ }^{\mathrm{c}}$ After diagnosis of advanced and metastatic disease but prior to initiation of novel therapy 
a

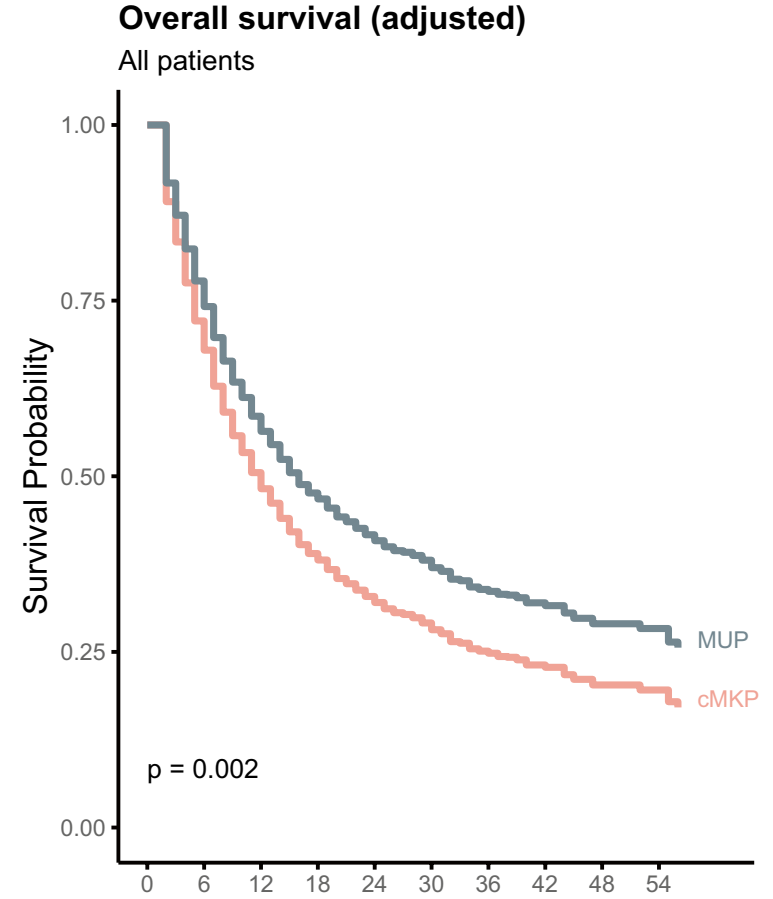

Time in months b Overall survival (adjusted)

Anti-PD1 therapy ever

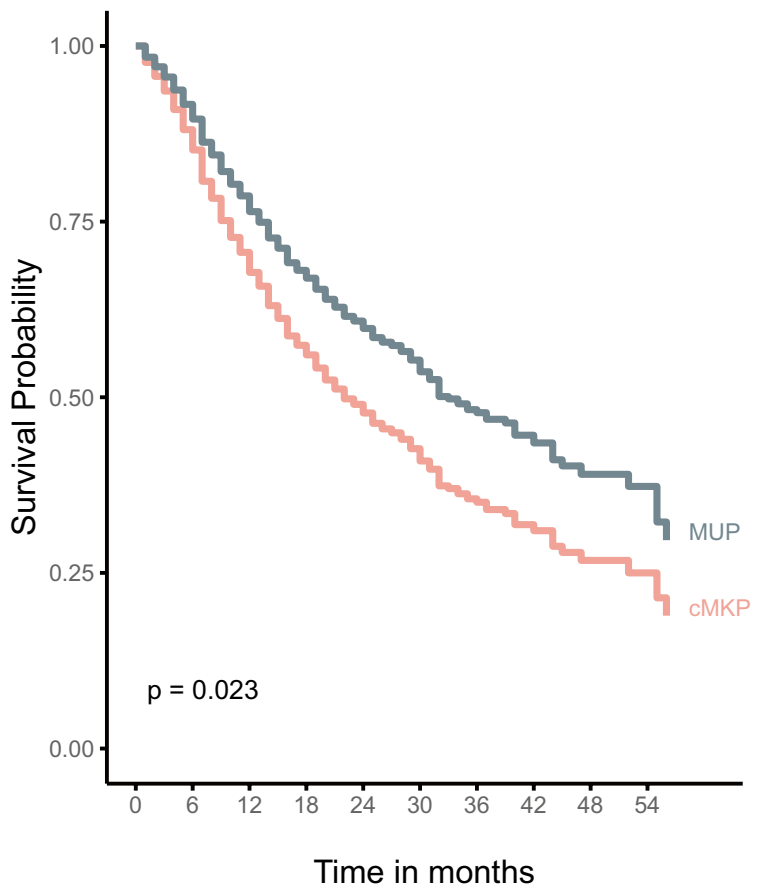

Fig. 4 Adjusted (expected) survival from start of novel therapy based on the multivariable cox models in $\mathbf{a}$ all patients and $\mathbf{b}$ patients ever treated with anti-PD1 therapy (monotherapy and combined)

Acknowledgements The authors thank all physicians and data managers who registered the patient data in the Dutch Melanoma Treatment Registry.

Funding This research was funded by The Netherlands Organisation for Health Research and Development (ZonMW), Grant Number 836002002 . This subsidy is part of the program of effectiveness research of high-cost medicine. The first four years (2012-2016) of the Dutch Melanoma Treatment Registry (DMTR) were sponsored by Roche Nederland B.V, Bristol-Myers Squibb (BMS), GlaxoSmithKline (GSK)/Novartis and, since 2015, also by Merck Sharp and Dohme (MSD).

Data accessibility The data that support the findings of this study are available from the Dutch Melanoma Treatment Registry. Restrictions apply to the availability of these data, which were used under licence for this study. Data are available from the corresponding author upon reasonable request with the permission of the Dutch Melanoma Treatment Registry.

\section{Compliance with ethical Standards}

Conflict of interest AvA has consultancy/advisory relationships with Amgen, Bristol-Myers Squibb, Novartis, MSD-Merck, Merck-Pfizer, Sanofi, 4SC and has received research grants not related to this paper from Amgen, Bristol-Myers Squibb, Novartis, Merck-Pfizer. MA has consultancy/advisory relationships with Pfizer, BMS, MERCK, Sanofi, Astellas, Pierre fabre, and Novartis. AvdE has advisory relationships with Amgen, Bristol-Myers Squibb, Roche, Novartis, MSD,
Pierre Fabre. JdG has advisory relationships with Bristol-Myers Squibb, Roche, Pierre Fabre, Servier, MSD, Novartis. JH has advisory relationships with Amgen, AstraZeneca, Bayer, Bristol-Myers Squibb, Celsius Therapeutics, GSK, Immunocore, Ipsen, MSD, Merck Serono, Novartis, Neon Therapeutics, Pfizer, Roche/Genentech, Sanofi, Seattle Genetics and has received research grants not related to this paper from Novartis, Bristol-Myers Squibb, MSD, Neon Therapeutics. GH consultancy/advisory relationships with Amgen, Bristol-Myers Squibb, Roche, MSD, Pfizer, Novartis and has received research grants not related to this paper from Bristol-Myers Squibb, Seerave. EK has consultancy/advisory relationships with Bristol-Myers Squibb, Novartis, Merck, Pierre Fabre and received research grants not related to this paper from Bristol-Myers Squibb. KS has advisory relationships with Bristol-Myers Squibb, Roche, Novartis, MSD, Pierre Fabre. AvdV has consultancy relationships with Bristol-Myers Squibb, MSD, Roche, Novartis, Pierre Fabre, Pfizer, Sanofi, Ipsen, Eisai. All grants were paid to the institutions and not related to this work. All remaining authors have declared no conflicts of interest.

Ethical approval All procedures performed in studies involving human participants were in accordance with the ethical standards of the institutional and/or national research committee and with the 1964 Declaration of HELSINKI and its later amendments or comparable ethical standards. This article does not contain any studies with animals performed by any of the authors.

Informed consent Informed consent was obtained from all individual participants included in the study. 
Open Access This article is licensed under a Creative Commons Attribution 4.0 International License, which permits use, sharing, adaptation, distribution and reproduction in any medium or format, as long as you give appropriate credit to the original author(s) and the source, provide a link to the Creative Commons licence, and indicate if changes were made. The images or other third party material in this article are included in the article's Creative Commons licence, unless indicated otherwise in a credit line to the material. If material is not included in the article's Creative Commons licence and your intended use is not permitted by statutory regulation or exceeds the permitted use, you will need to obtain permission directly from the copyright holder. To view a copy of this licence, visit http://creativecommons.org/licenses/by/4.0/.

\section{References}

1. Verver D, van der Veldt A, van Akkooi A, Verhoef C, Grunhagen DJ, Louwman WJ (2019) Treatment of melanoma of unknown primary in the era of immunotherapy and targeted therapy: a dutch population-based study. Int J Cancer. https:// doi.org/10.1002/ijc.32229

2. Gershenwald JE, Scolyer RA, Hess KR, Sondak VK, Long GV, Ross MI, Lazar AJ, Faries MB, Kirkwood JM, McArthur GA, Haydu LE, Eggermont AMM, et al. (2017) Melanoma staging: Evidence-based changes in the American Joint Committee on Cancer eighth edition cancer staging manual. CA Cancer J Clin. https://doi.org/10.3322/caac.21409

3. Song Y, Karakousis GC (2019) Melanoma of unknown primary. J Surg Oncol 119:232-241

4. Scott JF, Conic RZ, Thompson CL, Gerstenblith MR, Bordeaux JS (2018) Stage IV melanoma of unknown primary: a population-based study in the united states from 1973 to 2014. J Am Acad Dermatol. https://doi.org/10.1016/j.jaad.2018.03.021

5. Smith JL Jr, Stehlin JS Jr (1965) Spontaneous regression of primary malignant melanomas with regional metastases. Cancer 18:1399-1415

6. Giuliano AE, Moseley HS, Morton DL (1980) Clinical aspects of unknown primary melanoma. Ann Surg 191:98-104

7. Panagopoulos E, Murray D (1983) Metastatic malignant melanoma of unknown primary origin: a study of 30 cases. J Surg Oncol 23:8-10

8. Anbari KK, Schuchter LM, Bucky LP, Mick R, Synnestvedt M, Dt G, Hamilton R, Halpern AC (1997) Melanoma of unknown primary site: presentation, treatment, and prognosis-a single institution study. University of Pennsylvania Pigmented Lesion Study Group. Cancer 79:1816-1821

9. Savoia P, Fava P, Osella-Abate S, Nardo T, Comessatti A, Quaglino P, Bernengo MG (2010) Melanoma of unknown primary site: a 33-year experience at the Turin Melanoma Centre. Melanoma Res 20:227-232

10. Cervinkova M, Kucerova P, Cizkova J (2017) Spontaneous regression of malignant melanoma - is it based on the interplay between host immune system and melanoma antigens. Anticancer Drugs 28:819-830

11. Tefany FJ, Barnetson RS, Halliday GM, McCarthy SW, McCarthy WH (1991) Immunocytochemical analysis of the cellular infiltrate in primary regressing and non-regressing malignant melanoma. $\mathrm{J}$ Invest Dermatol 97:197-202

12. Saleh FH, Crotty KA, Hersey P, Menzies SW (2001) Primary melanoma tumour regression associated with an immune response to the tumour-associated antigen melan-A/MART-1. Int J Cancer 94:551-557
13. Aung PP, Nagarajan P, Prieto VG (2017) Regression in primary cutaneous melanoma: etiopathogenesis and clinical significance. Lab Invest. https://doi.org/10.1038/labinvest.2017.8

14. Bae JM, Choi YY, Kim DS, Lee JH, Jang HS, Lee JH, Kim H, Oh BH, Roh MR, Nam KA, Chung KY (2015) Metastatic melanomas of unknown primary show better prognosis than those of known primary: a systematic review and meta-analysis of observational studies. J Am Acad Dermatol 72:59-70

15. Rozeman EA, Dekker TJA, Haanen J, Blank CU (2018) Advanced melanoma: current treatment options, biomarkers, and future perspectives. Am J Clin Dermatol 19:303-317

16. Hodi FS, O'Day SJ, McDermott DF, Weber RW, Sosman JA, Haanen JB, Gonzalez R, Robert C, Schadendorf D, Hassel JC, Akerley W, van den Eertwegh AJ et al (2010) Improved survival with ipilimumab in patients with metastatic melanoma. N Engl J Med 363:711-723

17. Robert C, Thomas L, Bondarenko I, O'Day S, Weber J, Garbe C, Lebbe C, Baurain JF, Testori A, Grob JJ, Davidson N, Richards J et al (2011) Ipilimumab plus dacarbazine for previously untreated metastatic melanoma. N Engl J Med 364:2517-2526

18. Robert C, Long GV, Brady B, Dutriaux C, Maio M, Mortier L, Hassel JC, Rutkowski P, McNeil C, Kalinka-Warzocha E, Savage KJ, Hernberg MM et al (2015) Nivolumab in previously untreated melanoma without BRAF mutation. N Engl J Med 372:320-330

19. Robert C, Schachter J, Long GV, Arance A, Grob JJ, Mortier L, Daud A, Carlino MS, McNeil C, Lotem M, Larkin J, Lorigan $P$ et al (2015) Pembrolizumab versus Ipilimumab in Advanced Melanoma. N Engl J Med 372:2521-2532

20. Weber JS, D’Angelo SP, Minor D, Hodi FS, Gutzmer R, Neyns B, Hoeller C, Khushalani NI, Miller WH Jr, Lao CD, Linette GP, Thomas L et al (2015) Nivolumab versus chemotherapy in patients with advanced melanoma who progressed after anti-CTLA-4 treatment (CheckMate 037): a randomised, controlled, open-label, phase 3 trial. Lancet Oncol 16:375-384

21. Ribas A, Puzanov I, Dummer R, Schadendorf D, Hamid O, Robert C, Hodi FS, Schachter J, Pavlick AC, Lewis KD, Cranmer LD, Blank CU et al (2015) Pembrolizumab versus investigatorchoice chemotherapy for ipilimumab-refractory melanoma (KEYNOTE-002): a randomised, controlled, phase 2 trial. Lancet Oncol 16:908-918

22. Larkin J, Chiarion-Sileni V, Gonzalez R, Grob JJ, Cowey CL, Lao CD, Schadendorf D, Dummer R, Smylie M, Rutkowski P, Ferrucci PF, Hill A et al (2015) Combined Nivolumab and Ipilimumab or monotherapy in untreated melanoma. N Engl J Med 373:23-34

23. Chapman PB, Hauschild A, Robert C, Haanen JB, Ascierto P, Larkin J, Dummer R, Garbe C, Testori A, Maio M, Hogg D, Lorigan $P$ et al (2011) Improved survival with vemurafenib in melanoma with BRAF V600E mutation. N Engl J Med 364:2507-2516

24. Hauschild A, Grob JJ, Demidov LV, Jouary T, Gutzmer R, Millward M, Rutkowski P, Blank CU, Miller WH Jr, Kaempgen E, Martin-Algarra S, Karaszewska B et al (2012) Dabrafenib in BRAF-mutated metastatic melanoma: a multicentre, open-label, phase 3 randomised controlled trial. Lancet 380:358-365

25. Flaherty KT, Robert C, Hersey P, Nathan P, Garbe C, Milhem M, Demidov LV, Hassel JC, Rutkowski P, Mohr P, Dummer R, Trefzer $U$ et al (2012) Improved survival with MEK inhibition in BRAF-mutated melanoma. N Engl J Med 367:107-114

26. Long GV, Stroyakovskiy D, Gogas H, Levchenko E, de Braud F, Larkin J, Garbe C, Jouary T, Hauschild A, Grob JJ, ChiarionSileni V, Lebbe C et al (2015) Dabrafenib and trametinib versus dabrafenib and placebo for Val600 BRAF-mutant melanoma: a multicentre, double-blind, phase 3 randomised controlled trial. Lancet 386:444-451

27. Robert C, Karaszewska B, Schachter J, Rutkowski P, Mackiewicz A, Stroiakovski D, Lichinitser M, Dummer R, Grange F, Mortier L, Chiarion-Sileni V, Drucis K et al (2015) Improved overall 
survival in melanoma with combined dabrafenib and trametinib. N Engl J Med 372:30-39

28. Larkin J, Ascierto PA, Dreno B, Atkinson V, Liszkay G, Maio M, Mandala M, Demidov L, Stroyakovskiy D, Thomas L, de la Cruz-Merino L, Dutriaux C et al (2014) Combined vemurafenib and cobimetinib in BRAF-mutated melanoma. N Engl J Med 371:1867-1876

29. Dummer R, Ascierto PA, Gogas HJ, Arance A, Mandala M, Liszkay G, Garbe C, Schadendorf D, Krajsova I, Gutzmer R, ChiarionSileni V, Dutriaux C et al (2018) Encorafenib plus binimetinib versus vemurafenib or encorafenib in patients with BRAF-mutant melanoma (COLUMBUS): a multicentre, open-label, randomised phase 3 trial. Lancet Oncol 19:603-615

30. Ascierto PA, Dummer R (2018) Immunological effects of BRAF+MEK inhibition. Oncoimmunology 7:e1468955

31. Jochems A, Schouwenburg MG, Leeneman B, Franken MG, van den Eertwegh AJ, Haanen JB, Gelderblom H, Uyl-de Groot CA, Aarts MJ, van den Berkmortel FW, Blokx WA, Cardous-Ubbink MC et al (2017) Dutch melanoma treatment registry: quality assurance in the care of patients with metastatic melanoma in the Netherlands. Eur J Cancer 72:156-165

32. van Zeijl MCT, de Wreede LC, van den Eertwegh AJM, Wouters M, Jochems A, Schouwenburg MG, Aarts MJB, van Akkooi ACJ, van den Berkmortel F, de Groot JWB, Hospers GAP, Kapiteijn E et al (2020) Survival outcomes of patients with advanced melanoma from 2013 to 2017: Results of a nationwide populationbased registry. Eur J Cancer 144:242-251

33. Manola J, Atkins M, Ibrahim J, Kirkwood J (2000) Prognostic factors in metastatic melanoma: a pooled analysis of eastern cooperative oncology group trials. J Clin Oncol 18:3782-3793

34. van Zeijl MCT, Ismail RK, de Wreede LC, van den Eertwegh AJM, de Boer A, van Dartel M, Hilarius DL, Aarts MJB, van den Berkmortel F, Boers-Sonderen MJ, de Groot JB, Hospers GAP et al (2020) Real-world outcomes of advanced melanoma patients not represented in phase III trials. Int J Cancer 147:3461-3470

35. Scott JF, Gerstenblith MR. (2018) Noncutaneous Melanoma. Melanoma of Unknown Primary, Codon Publications, Singapore

36. Luen S, Wong SW, Mar V, Kelly JW, McLean C, McArthur GA, Haydon A (2018) Primary tumor thickness is a prognostic factor in stage iv melanoma: a retrospective study of primary tumor characteristics. Am J Clin Oncol 41:90-94
37. Bedikian AY, Johnson MM, Warneke CL, Papadopoulos NE, Kim K, Hwu WJ, McIntyre S, Hwu P (2008) Prognostic factors that determine the long-term survival of patients with unresectable metastatic melanoma. Cancer Invest 26:624-633

38. Lee CC, Faries MB, Wanek LA, Morton DL (2009) Improved survival for stage IV melanoma from an unknown primary site. $\mathrm{J}$ Clin Oncol 27:3489-3495

39. Ellebaek E, Bastholt L, Schmidt H, Svane IM, Donia M (2019) The real-world outcome of metastatic melanoma: unknown primary vs. known cutaneous. Int J Cancer 145:3173-3174

40. Verver D, van der Veldt AAM, van Akkooi ACJ, Verhoef K, Grunhagen DJ, Louwman MWJ (2019) Author's reply to: the realworld outcome of metastatic melanoma: unknown primary vs. known cutaneous. Int J Cancer 145:3175-3176

41. Gambichler T, Chatzipantazi M, Schröter U, Stockfleth E, Gedik C (2019) Patients with melanoma of unknown primary show better outcome under immune checkpoint inhibitor therapy than patients with known primary: preliminary results. OncoImmunology 8:1677139

42. Dasgupta T, Bowden L, Berg JW (1963) Malignant melanoma of unknown primary origin. Surg Gynecol Obstet 117:341-345

43. Egberts F, Bergner I, Kruger S, Haag J, Behrens HM, Hauschild A, Rocken C (2014) Metastatic melanoma of unknown primary resembles the genotype of cutaneous melanomas. Ann Oncol 25:246-250

44. Dutton-Regester K, Kakavand H, Aoude LG, Stark MS, Gartside MG, Johansson P, O'Connor L, Lanagan C, Tembe V, Pupo GM, Haydu LE, Schmidt CW et al (2013) Melanomas of unknown primary have a mutation profile consistent with cutaneous sunexposed melanoma. Pigment Cell Melanoma Res 26:852-860

45. Gos A, Jurkowska M, van Akkooi A, Robert C, Kosela-Paterczyk H, Koljenovic S, Kamsukom N, Michej W, Jeziorski A, Pluta P, Verhoef C, Siedlecki JA et al (2014) Molecular characterization and patient outcome of melanoma nodal metastases and an unknown primary site. Ann Surg Oncol 21:4317-4323

Publisher's Note Springer Nature remains neutral with regard to jurisdictional claims in published maps and institutional affiliations.

\title{
Authors and Affiliations
}

\author{
Danielle Verver ${ }^{1}$ (1) Dirk J. Grünhagen ${ }^{1}$. Alexander C. J. van Akkooi ${ }^{2} \cdot$ Maureen J. B. Aarts $^{3}$. \\ Franchette W. P. J. van den Berkmortel ${ }^{4}$. Alfonsus J. M. van den Eertwegh ${ }^{5}$. Jan Willem B. de Groot ${ }^{6}$. \\ Marye J. Boers-Sonderen ${ }^{7}$. John B. A. G. Haanen ${ }^{8}$. Geke A. P. Hospers ${ }^{9}$. Ellen Kapiteijn $^{10}$. Djura Piersma ${ }^{11}$. \\ Rozemarijn S. van Rijn ${ }^{12} \cdot$ Karijn P. M. Suijkerbuijk ${ }^{13}$. Albert J.ten Tije ${ }^{14} \cdot$ Gerard Vreugdenhil $^{15} \cdot$ Cornelis Verhoef $^{1}$. \\ Astrid A. M. van der Veldt ${ }^{16}$
}

1 Department of Surgical Oncology, Erasmus MC Cancer Institute, Dr Molewaterplein 40, 3015 GD Rotterdam, The Netherlands

2 Department of Surgical Oncology, Netherlands Cancer Institute-Antoni van Leeuwenhoek, Amsterdam, The Netherlands

3 Department of Medical Oncology, Maastricht University Medical Centre+, Maastricht, The Netherlands

4 Department of Medical Oncology, Zuyderland Medical Centre, Sittard-Geleen, The Netherlands
5 Department of Medical Oncology, Amsterdam UMC, Location VU University Medical Centre (VUmc), Cancer Centre Amsterdam, Amsterdam, The Netherlands

6 Oncology Centre Isala, Isala, Zwolle, The Netherlands

7 Department of Medical Oncology, Radboud University Medical Centre, Nijmegen, The Netherlands

8 Department of Medical Oncology, Netherlands Cancer Institute, Amsterdam, The Netherlands 
9 Department of Medical Oncology, University Medical Centre Groningen, University of Groningen, Groningen, The Netherlands

10 Department of Medical Oncology, Leiden University Medical Centre, Leiden, The Netherlands

11 Department of Internal Medicine, Medisch Spectrum Twente, Enschede, The Netherlands

12 Department of Internal Medicine, Medical Centre Leeuwarden, Leeuwarden, The Netherlands
13 Department of Medical Oncology, University Medical Centre Utrecht Cancer Centre, Utrecht, The Netherlands

14 Department of Internal Medicine, Amphia Hospital, Breda, The Netherlands

15 Department of Internal Medicine, Maxima Medical Centre, Eindhoven, The Netherlands

16 Departments of Medical Oncology and Radiology and Nuclear Medicine, Erasmus MC Cancer Institute, Rotterdam, The Netherlands 